\title{
In-pipe mobile robot with passive adaptation ability to pipe diameter
}

\author{
Mohammad Reza Elhami, Iman Dashti
}

\begin{abstract}
In-pipe robotic systems are increasingly being used for visual inspection and nondestructive testing to monitor block, corrosion, crack, defect, and wall thickness of pipeline networks. In this paper an in-pipe mobile robot with the passive ability of adaptation to pipe diameter is presented. Its mechanical structure consists of three sets of parallelogram wheeled leg mechanism which are circumferentially spaced out 120 degrees apart symmetrically. Using a simple preloaded spring makes this structural design capable to realize the adaptation to a wide range of pipe diameters, from 20 to 32 inch, and also wheel normal forces adjustment. On the basis of analyzing the mechanical actions of the adaptation to pipe diameter and normal force adjusting, the related mechanical models are derived. Using simulations performed by Msc Adams the derived models are validated and also the ability of robot in performing challenging tasks in different pipe profiles is inspected. Dynamic simulations prove that the robot can pass well through reducer joints and vertical pipelines by adjusting the spring force such that the suitable thrust force for the robot is obtained.
\end{abstract}

Keywords-in-pipe robot, passive pipe diameter adaptability, normal force adjusting, pipelines inspection

\section{Introduction}

Pipeline networks are often exposed to the risk of block and leakage. Furthermore, since most urban gas pipelines are buried under the ground, cracks and damages in the welded region of pipelines may be caused by the third party such as construction, electricity, and sewage project. Therefore, inspection, maintenance and repair for gas pipeline are needed strongly. Inspection for pipelines is a challenging task because of the complex internal geometry and hazard contents of pipes. Therefore, employing robots that can run through inside pipelines to perform inspection and maintenance seems to be an effective solution.

In-pipe robots can be classified into several elementary forms according to movement patterns, as shown in Fig. 1 and Fig. 2. As shown in Fig. 2(a), for example, the pig type is one of the most well-known commercial ones, which is passively driven by the fluid pressure inside pipelines. It has been employed for the inspection of pipelines with large diameters [1]. The wheel type illustrated in Fig. 2(b) is similar to the plain mobile robot, and a number of commercialized robots have been reported up to now [2], [3]. Fig. 2(c) shows the robot with caterpillars instead of wheels [4]. As shown in Fig. 2(d), the wall-press type, which has a number of advantages in climbing vertical pipelines, corresponds to the robot with a flexible mechanism for pressing the wall with whatever means they apply [5], [6].

Mohammad Reza Elhami, Assoc. Prof., Iman Dashti, MEng.

Imam Hosein University, Iran
As depicted in Fig. 2(e), the walking type possessing articulated legs can produce highly sophisticated motions [7], [8]. The inchworm type given in Fig. 2(f) is usually employed for pipelines with very small diameters [9], [10]. The screw type (or helical-drive type) displays the motion of a screw when it advances in the pipelines, as depicted in Fig. 2(g) [11], [12].

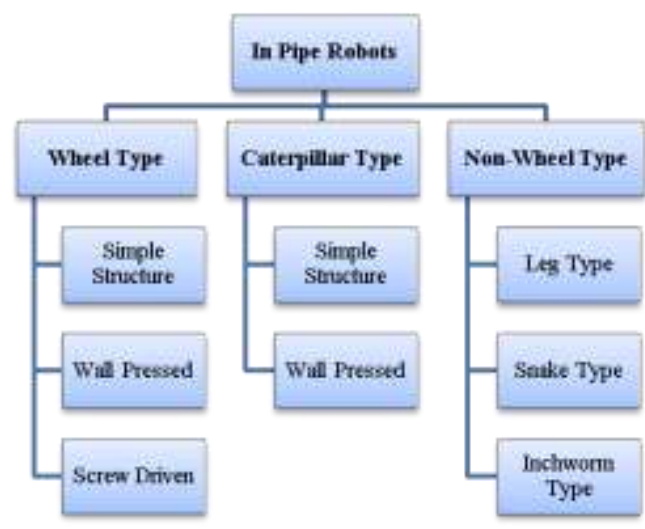

Figure 1. Classification of in-pipe robots

Although an in-pipe robotic system is in theory very useful, employing it for such an environment requires innovative development and integration of various technologies in the aspect of mechanism design, as well as intelligent control architecture. Two main requirements in mechanism design of an in-pipe robot are 1) Flexibility to change body depending on shape, size and the configuration of the pipelines. 2) Providing sufficient tractive force to pull equipment, climb on vertical pipelines and go over obstacles. In this paper a mechanism is proposed for an inpipe robot to meet these requirements and its performance is analyzed in most challenging pipe configurations i.e. reducer and vertical pipe.

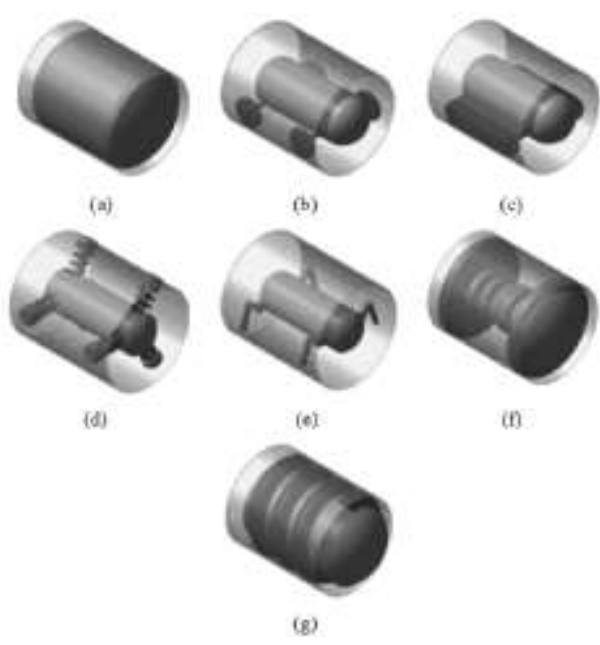

Figure 2. In-pipe robot motion mechanisms 


\section{Overview of Mechanism}

One of the most important issues in the design of a driving vehicle is how to obtain the traction force enough to pull instrumentation as well as the vehicle itself. Especially in vertical pipelines, it is desirable to keep adequate wall pressing forces in order to ensure sufficient traction forces. Excessive forces may dissipate power and be in danger of damaging the robot. On the contrary insufficient forces may cause the robot to fall down. On the condition that the wheel does not slip on the pipeline surfaces, the traction force is proportional to the friction coefficient and the pressing force between the wheel and the pipeline surface, and the friction coefficient depends on the material of wheel and the surface condition of pipelines. In addition, the linkage mechanism of the vehicle should minimize the variation of traction force caused by variation of pipeline diameters. Therefore, a leg mechanism has to meet the following three requirements. At first, it should be possible to push against the pipeline wall with adequate pressing forces. In the second, the pressing force should not show significant change during navigation in order to provide stable traction force and flexible locomotion. At last, the mechanism should be simple and small in size to occupy minimal space inside the pipelines.

The pipe diameter adaptive mechanism presented here is composed of three sets of parallelogram wheeled legs circumferentially spaced out 120 degrees apart symmetrically. Each parallelogram wheeled leg has two driving wheels in front and rear.
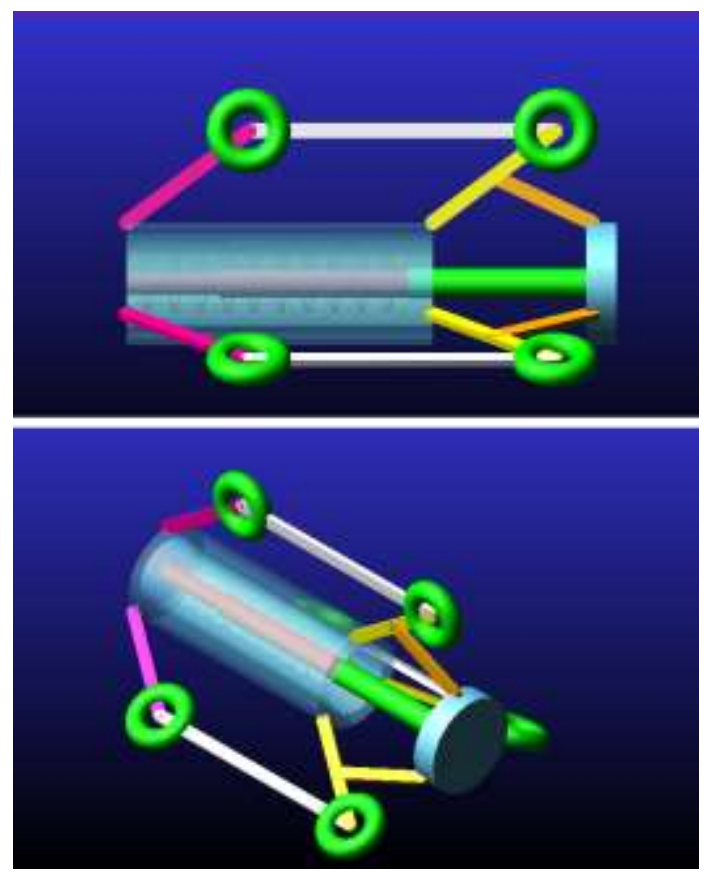

Figure 3. Robot with adaptation mechanism

The parallelogram mechanisms are connected to chassis and a sliding bush by revolute joints. The bush is connected to an adjustable spring and slides through chassis that permits natural folding and unfolding of the legs. The spring presses the sliding bush which can push three sets of parallelogram wheeled legs to make driving wheels contact to inner wall of pipe, or adjust the pressure between driving wheels and pipe wall. This structural design makes it possible to realize the adaptability to pipe diameter and tractive force adjusting together. With this structure adjustment to pipe diameter can be realized in a wide range from 480 to $820 \mathrm{~mm}$.

\section{Modeling of Normal Force Adjustment}

In this section we establish a mechanical model for normal force adjustment on the basis of analyzing relationships among normal forces, additional pressure and spring force. We define the sum of all pressures applied to driving wheels by the robot weight as total supporting force denoted by symbol $\sum \mathrm{N}$ and the sum of pressures produced by the spring force mechanism as additional pressure denoted by symbol $\sum \mathrm{P}$. Since three sets of parallelogram wheeled legs are circumferentially spaced out 120 degrees

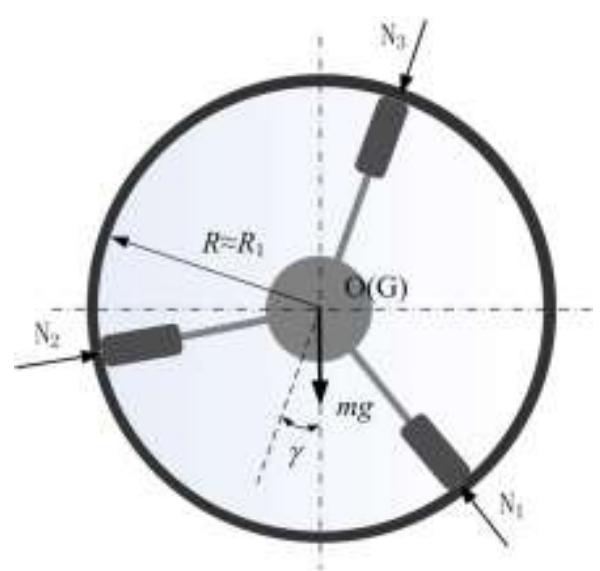

Figure 4. Normal force distribution

apart symmetrically, any attitude angle of the robot will result that only one or two sets of driving wheel at the bottom are contributors which support the gravity of the robot. In Fig. $4, \mathrm{~N}_{1}, \mathrm{~N}_{2}$ and $\mathrm{N}_{3}$ are used to denote the supporting force applied to three sets of driving wheels respectively. We define that a counter-clockwise attitude angle, $\gamma$, is positive, and a clockwise angle is negative. Thus we have

$$
\left\{\begin{array}{l}
N_{3}=0 ;-60 \leq \gamma \leq 60 \\
N_{1}=0 ; 60 \leq \gamma \leq 180 \\
N_{2}=0 ; 180 \leq \gamma \leq 300
\end{array}\right.
$$

From equilibrium of forces we have:

$$
\begin{aligned}
& \left\{\begin{array}{l}
N_{1} \cos (\gamma+60)+N_{2} \cos (60-\gamma)=m g \\
N_{1} \sin (\gamma+60)-N_{2} \sin (60-\gamma)=0
\end{array} ;-60 \leq \gamma \leq 60\right. \\
& \left\{\begin{array}{l}
N_{2} \cos (\gamma-60)-N_{3} \cos (\gamma)=m g \\
N_{2} \sin (\gamma-60)-N_{3} \sin (\gamma)=0
\end{array} ; 60 \leq \gamma \leq 180\right.
\end{aligned}
$$




$$
\left\{\begin{array}{l}
-N_{3} \cos (\gamma)+N_{1} \cos (60+\gamma)=m g \\
-N_{3} \sin (\gamma)+N_{1} \sin (60+\gamma)=0
\end{array} ; 180 \leq \gamma \leq 300\right.
$$

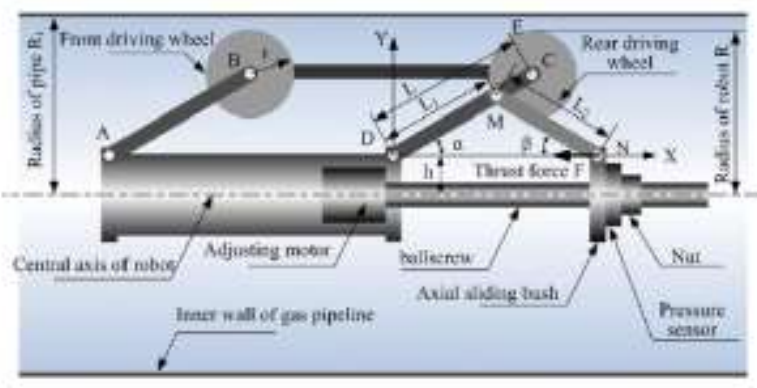

Figure 5. Robot dimensional nomenclature

Solving above equations and considering slope angle, $\varphi$, we obtain:

$$
\sum N=\left\{\begin{array}{l}
2 m g \cos \gamma \cos \varphi ;-60 \leq \gamma \leq 60 \\
2 m g \cos (\gamma-120) \cos \varphi ; 60 \leq \gamma \leq 180 \\
2 m g \cos (\gamma-240) \cos \varphi ; 180 \leq \gamma \leq 300
\end{array}\right.
$$

The following geometric relationships are extracted from Fig. 5:

$$
\left\{\begin{array}{l}
R=r+h+L \sin \alpha \\
y=R-h \\
x=L_{1} \cos \alpha+L_{2} \cos \beta \\
L_{1} \sin \alpha=L_{2} \sin \beta
\end{array}\right.
$$

According to virtual work principle:

$$
\left(\sum N+\sum P\right) d y+F d x=0
$$

By differentiating (6) and substituting the results in (7) we obtain:

$$
F=\frac{L}{L_{1}(R-r-h)\left(\frac{1}{\sqrt{L^{2}-(R-r-h)^{2}}}+\frac{L_{1}}{\sqrt{L^{2} L_{2}^{2}-L_{1}^{2}(R-r-h)^{2}}}\right)}\left(\sum N+\sum P\right)
$$

Equation 9 obtains the required spring force ,F , for the desired additional normal force $\sum \mathrm{P}$.

If the driving force of motion of motor is sufficient the relationship between normal force and thrust force for the robot will be as:

$$
F_{T}=\left(\sum N+\sum P\right) \mu
$$

Using (9) and (10) we can set the spring force value so that the required thrust force is obtained for the robot to accomplish its mission.

\section{Simulation and Results}

The dynamic model of robot was simulated by MSC Adams to analyze robot performance. Using Adams Evaluation Tool, the mechanical model of normal force adjustment was analyzed and the relationship between spring preload and total normal force on wheels was extracted. Simulations are performed in a 650 (mm) diameter pipe and Results are shown in Fig. 6. The solid line illustrates the results from mechanical model and dashed line presents dynamic model results in Adams. As can be seen in both curves total normal force increases by increasing spring preload and there is a good analogues between the two curves.

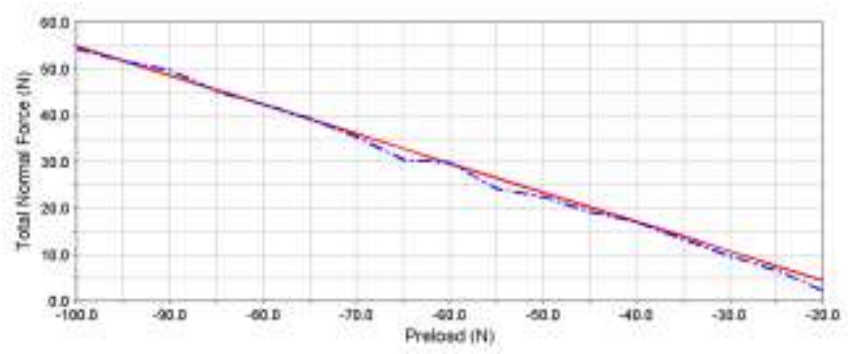

Figure 6. Spring force-Normal force curve

Here the robot performance is simulated in passing through a reducer. The operating range of pipe diameter for robot motion is between 20-30 (inch)., so from the standard dimensions of reducers according to ASME B16.9, standard of fittings dimensions, two reducers are selected to change the pipe diameter from the minimum value (20 inch) to maximum value (32 inch) and vise-versa (B32-24 and B2420). Fig. 7 shows the reducer designed with these dimensions and figs. 8-11 show the robot moving through pipe sections successfully. 
Proc. of the International Conference on Advances in Mechanical and Automation Engineering - MAE 2016

Copyright (c) Institute of Research Engineers and Doctors. All rights reserved.

ISBN: 978-1-63248-102-3 doi: 10.15224/ 978-1-63248-102-3-40

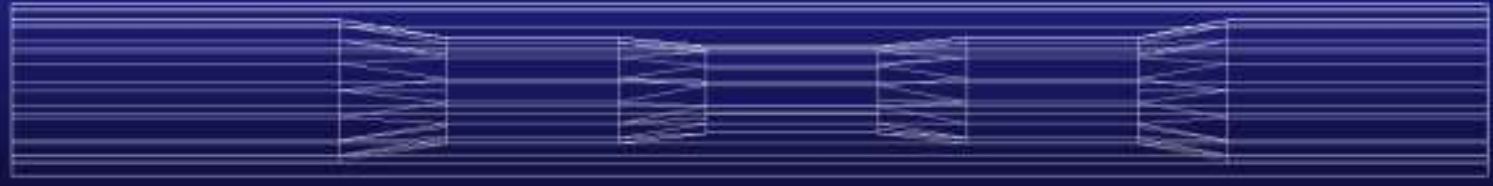

Figure 7. Reducer testbed

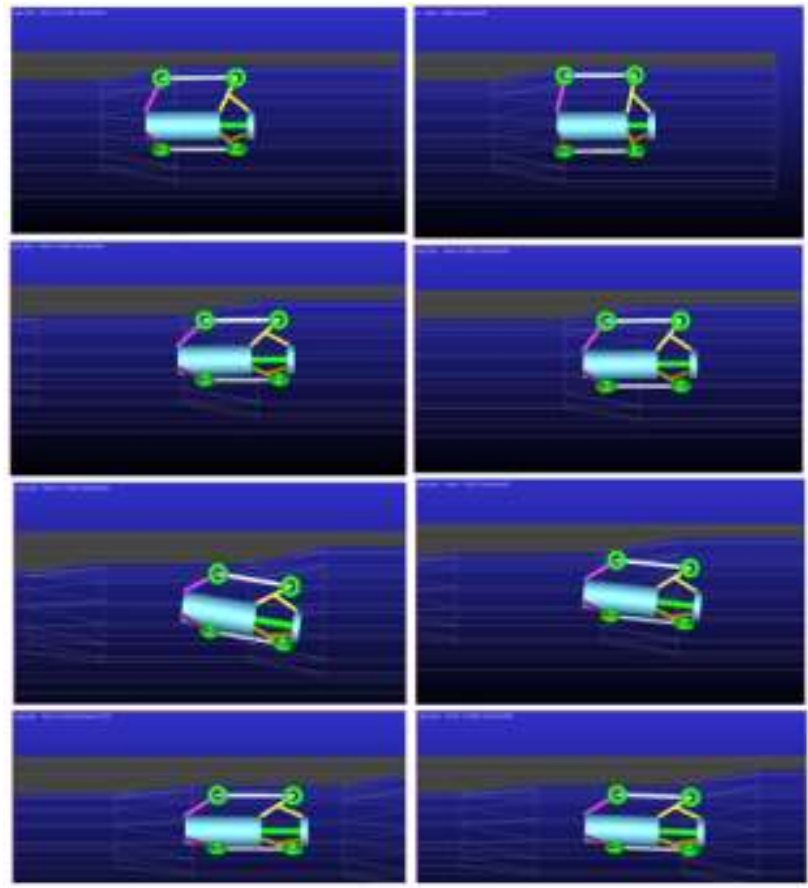

Figure 8. Robot passing through the first reducer

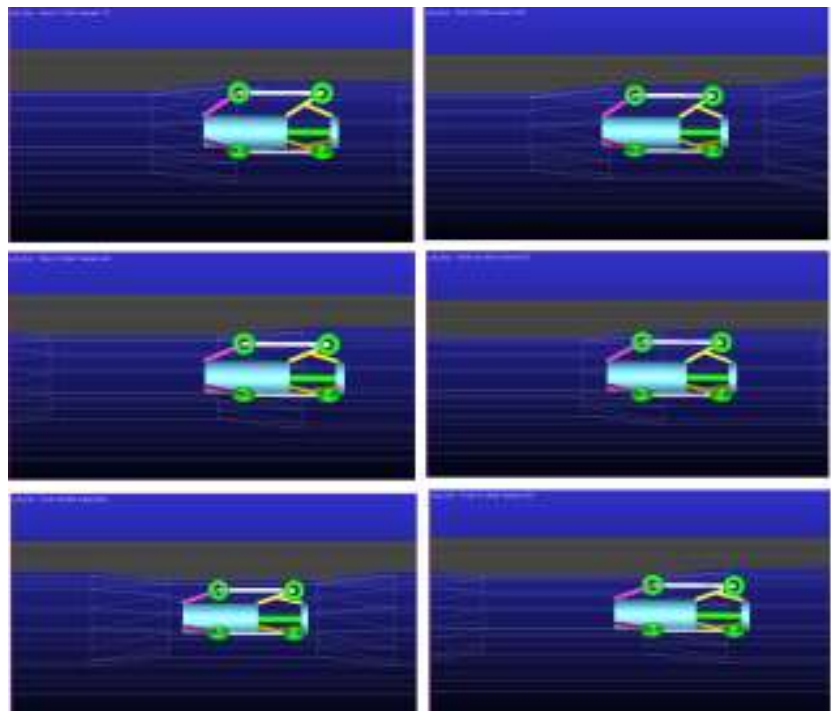

Figure 9. Robot passing through the second reducer

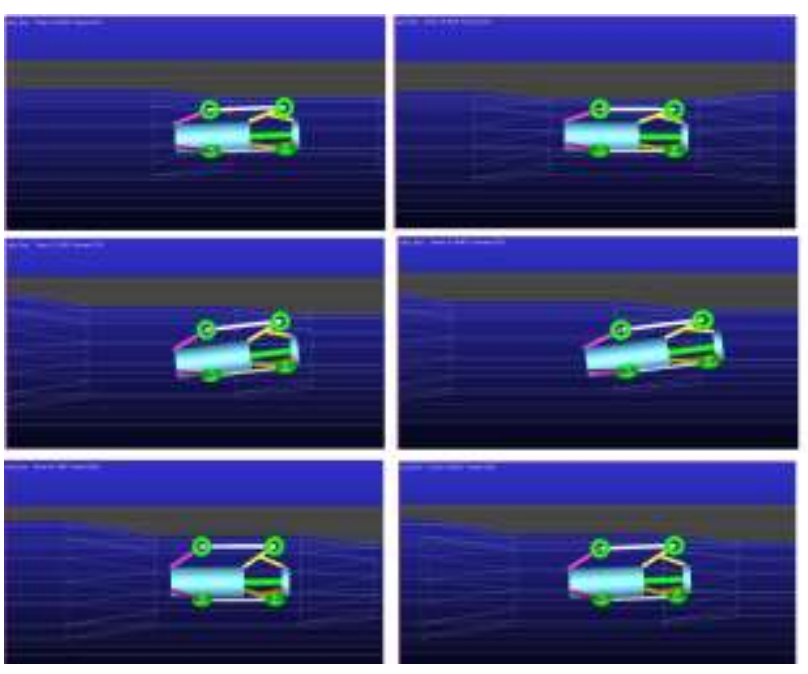

Figure 10. Robot passing through the third reducer

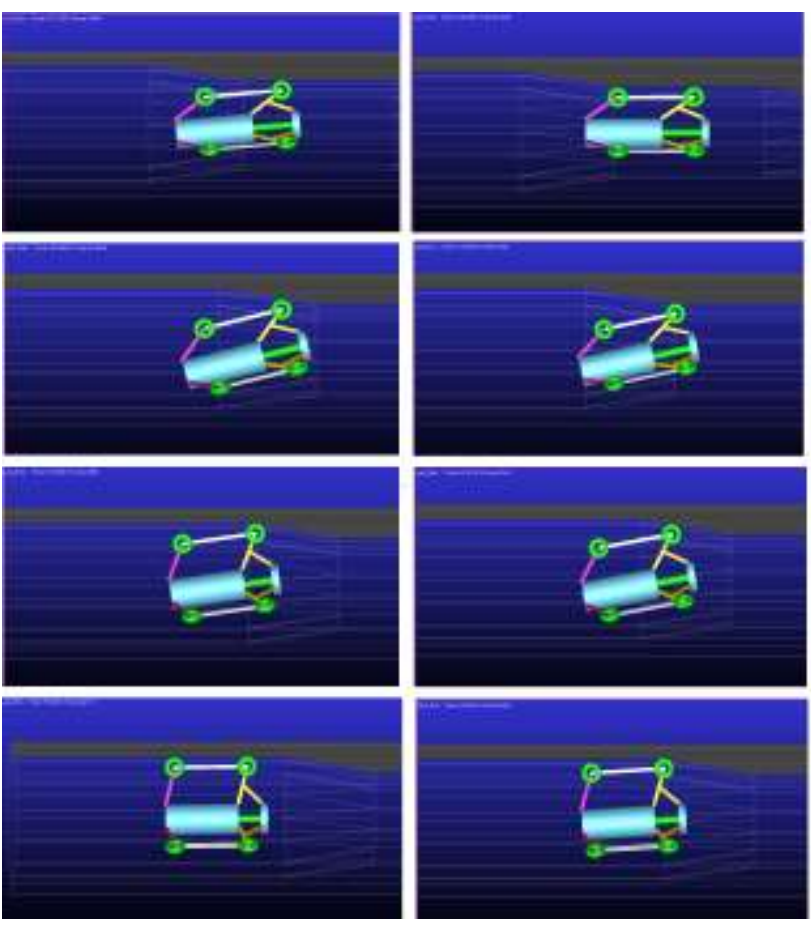

Figure 11. Robot passing through the forth reducer 
Robot was then simulated in a bent pipe with 650 $(\mathrm{mm})$ diameter and a curvature radius of $650(\mathrm{~mm})$ that bends toward vertical direction. As can be seen in Fig. 9 the robot could pass a $90(\mathrm{deg})$ bent and then climb the vertical section of the pipe successfully.
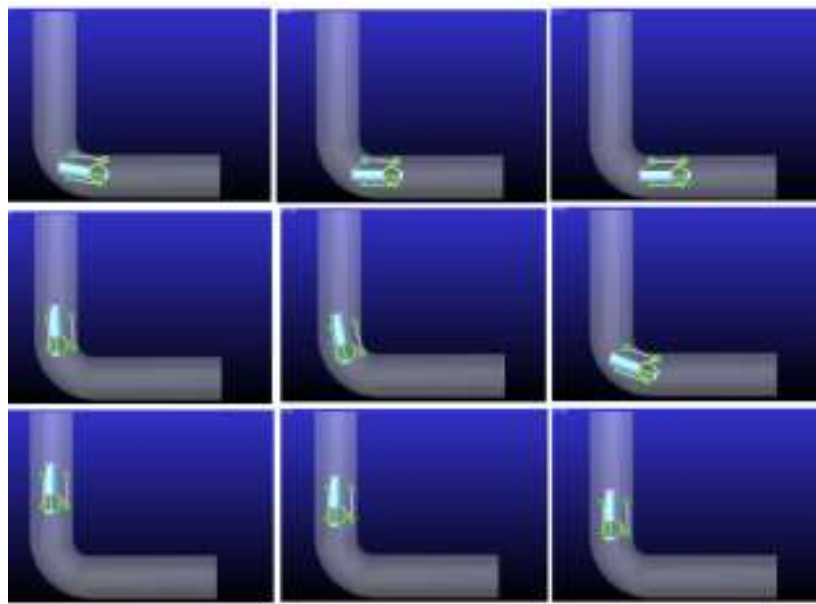

Figure 12. Robot in vertical bent

In previous section relationship between spring force and thrust force was obtained. In the case of a vertical pipe the minimum required thrust force in the vertical section is equal to robot weight. The mechanical model predicts the necessary spring force to provide such thrust force as 21.4 $(\mathrm{N})$. Lower than this value robot cannot provide sufficient thrust force to overcome the gravity force and complete the mission. Fig. 10 shows the robot velocity for two spring preload cases. The red curve is $22(\mathrm{~N})$ preload case and blue curve is for $20(\mathrm{~N})$ preload case. The results confirm that the required spring force for the robot obtained from mechanical model; as the minimum spring preload by which the robot succeeded to cross the pipe was 22 (N) and for case of $20(\mathrm{~N})$ robot just slips down the pipe and velocity turns to negative.

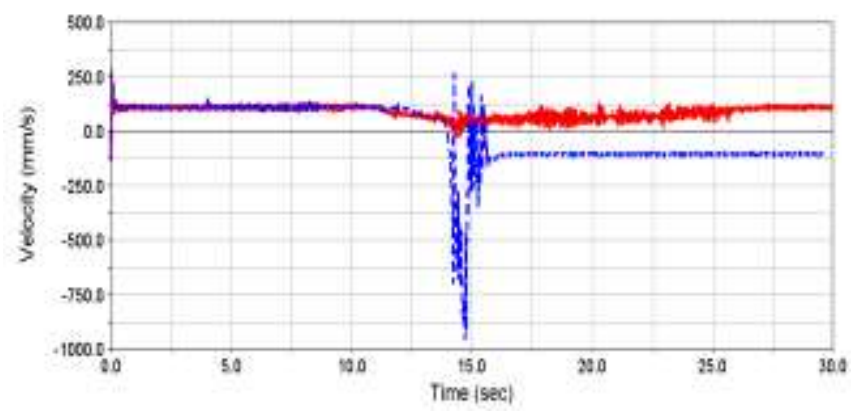

Figure 9. Robot velocity in vertical bent

\section{v. Conclusion}

This paper presents an in-pipe robot mechanism with simple structure and passive adaptability to shape and size of pipe in a wide range of diameters from $480 \mathrm{~mm}$ to 820 (mm). The spring force presses the wheels to inner pipe wall and allows for normal force adjustment to provide necessary thrust force in various conditions. A mechanical model was obtained based on relationship between spring force and normal forces on wheels. Dynamic simulations prove that the robot can pass well through reducer joints and vertical pipelines by adjusting the spring force such that the suitable thrust force for the robot is obtained.

\section{References}

[1] J. Okamoto, Jr., J. C. Adamowski, M. S. G. Tsuzuki, F. Buiochi, and $\mathrm{C}$.

S. Camerini, "Autonomous system for oil pipelines inspection," Mechatronics,

$$
\text { vol. 9, pp. 731-743, } 1999 .
$$

[2] H. Schempf, "GRISLEE: Gas Main Repair and Inspection System for

Live-Entry Environments," Gas Res. Inst., Doc. GRI-02/0132, 2002.

[3] S. G. Roh, S. M. Ryew, J. H. Yang, and H. R. Choi, "Actively steerable

inpipe inspection robots for underground urban gas pipelines," in

Proc.

IEEE Int. Conf. Robotics, Automation, 2001, pp. 761-766.

[4] H. T. Roman, B. A. Pellegrino, and W. R. Sigrist, "Pipe crawling inspection

robots: An overview," IEEE Trans. Energy Convers., vol. 8, pp.

576-583, Sept. 1993.

[5] S. Nagano and Y. Oka, "Application of in-pipe visual inspection robot

to piping internal surface lining," in Proc. 5th Int. Symp. Robotics in Construction, 1988, pp. 897-906.

[6] S. M. Ryew, S. H. Baik, S. W. Ryu, K. M. Jung, S. G. Roh, and H. R. Choi, "Inpipe inspection robot system with active steering mechanism,"

in Proc. IEEE Int. Conf. Intelligent Robots, Systems, 2000, pp. $1652-1657$.

[7] W. Neubauer, "A spider-like robot that climbs vertically in ducts or pipes," in Proc. IEEE/RSJ Int. Conf. Intelligent Robots, Systems, 1994, pp. $1178-1185$.

[8] F. Pfeiffer, T. Robmann, and K. Loffer, "Control of a tube crawling machine,"

in Proc. Int. Conf. Control of Oscillations and Chaos, vol. 3 , 2000, pp. 586-591.

[9] A. M. Bertetto and M. Ruggiu, "In-pipe inch-worm pneumatic flexible

robot," in Proc. IEEE/ASME Int. Conf. Advanced Intelligent Mechatronics, vol. 2, 2001, pp. 1226-1231.

[10] A. Menciassi, J. H. Park, S. Lee, S. Gorinil, P. Dario, and J.-O. Park,

"Robotic solutions and mechanisms for a semi-autonomous endoscope,"

in Proc. IEEE/RSJ Int. Conf. Intelligent Robots, Systems, 2002, pp.

1379-1384.

[11] I. Hayashi, N. Iwatsuki, and S. Iwashina, "The running characteristics

of a screw-principle microrobot in a small bent pipe," in Proc. Int. Symp.

Micro Machines, Human Science, 1995, pp. 225-228.

[12] M. Horodinca, I. Dorftei, E. Mignon, and A. Preumont, "A simple architecture for in-pipe inspection robots," in Proc. Int. Colloq. Mobile, Autonomous Systems, 2002, pp. 61-64. 